\title{
Listeriosis outbreak caused by Latin-style fresh cheese, Bizkaia, Spain, August 2012
}

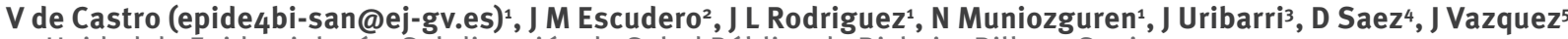

1. Unidad de Epidemiología, Subdirección de Salud Pública de Bizkaia, Bilbao, Spain

2. Unidad de Sanidad Alimentaria, Subdirección de Salud Pública de Bizkaia, Bilbao, Spain

3. Subárea de Seguridad Alimentaria y Zoonosis del Ayuntamiento de Bilbao, Bizkaia, Spain

4. Laboratorio Municipal de Bilbao, Bizkaia, Spain

5. Laboratorio de Referencia de Listeria, Instituto de Salud Carlos III, Madrid, Spain

Citation style for this article:

de Castro V, Escudero JM, Rodriguez JL, Muniozguren N, Uribarri J, Saez D, Vazquez J. Listeriosis outbreak caused by Latin-style fresh cheese, Bizkaia, Spain, August 2012. Euro Surveill. 2012;17(42):pii=20298. Available online: http://www.eurosurveillance.org/ViewArticle.aspx?Articleld=20298

Two cases of laboratory-confirmed listeriosis were detected in Bizkaia, Spain, at the end of August. The epidemiological investigation indicated that these two cases were associated with the consumption of Latin-style fresh cheese made from pasteurised milk in Portugal. Different batches of the same cheese were analysed and confirmed as contaminated with Listeria monocytogenes. The product was withdrawn from the market and the population was advised not to consume this kind of cheese.

Two cases of pregnancy-related listeriosis were detected in Bizkaia, Spain, between 28 and 30 August 2012: a pregnant woman and a newborn. The epidemiological investigation indicated that the pregnant woman and the mother of the newborn had consumed the same type of fresh cheese during the two months before symptom onset.

Listeriosis is a bacterial food-borne illness that can also be transmitted from the mother to the foetus during pregnancy or at birth. Possible symptoms of the illness in newborns and adults are meningoencephalitis, septicaemia, or both; it can also lead to abortion during pregnancy [1]. Listeriosis is an uncommon illness but quite severe. Therefore, it is important to be extremely careful with the food consumption behaviours of certain risk groups [2].

In Bizkaia (a territory of the Basque Country, in the north of Spain, with a population of nearly $1,150,000$ inhabitants), the incidence rate of listeriosis in the last ten years has been variable, between 0.88 and 2.11 per 100,000 inhabitants, with a mean of 15 cases per year (range: 7-23 cases) [3].

During 2012, as of 30 September, 10 cases of listeriosis have been notified in Bizkaia. Of these, meningoencephalitis has been identified in three cases, septicaemia in another three and mother-foetal transmission in the other four cases. The mortality rate recorded in 2012 has been of $30 \%$.

Of the ten cases of listeriosis investigated in 2012 in Bizkaia, the first eight were sporadic cases whereas the routine epidemiological investigation of the last two cases who occurred in August revealed the consumption of a particular kind of cheese (Latin-style fresh cheese) as mentioned above.

\section{Epidemiological investigation}

After the detection of the two cases in August, epidemiological questionnaires were carried out. The two cases were interviewed about their food consumption during the incubation period (two months before onset of the symptoms). For this outbreak, a listeriosis case was defined as an individual with clinically-compatible illness, from whom Listeria monocytogenes was isolated from a normally sterile site and who had consumed a Latin-style fresh cheese two months before symptom onset. This soft cheese, made from pasteurised milk in Portugal was mostly commercialised in shops selling Latin specialities, which are present almost in all the Spanish autonomous communities as well as in Italy and Portugal.

On 12 September, all listeriosis cases who had been notified in $2012(n=10)$ were contacted and asked specifically about the consumption of the Latin-style fresh cheese, the dates they had consumed it and the shop where they used to buy the cheese. There were no cheese leftovers in any of the patients' homes.

\section{Results}

The index case detected on 28 August 2012 was a 36 year-old woman with a 35 -week twin pregnancy. She was hospitalised on 24 August with fever $\left(39^{\circ} \mathrm{C}\right)$ that had lasted for three days. L. monocytogenes was isolated in blood culture and the strain was characterised as $1 / 2$ a serotype. The epidemiological investigation revealed the consumption of Latin-style fresh cheese 
bought in shop A, two months before the onset of symptoms.

The second case was a hospitalised newborn baby who developed sepsis at birth. Listeria was isolated in the blood culture of the baby and in the placenta and the strain was characterised as $1 / 2$ a serotype. The epidemiological investigation revealed that his mother had consumed Latin-style fresh cheese acquired in shop B, one month and a half before giving birth.

The evolution of the two patients was favourable. The first case gave birth to two healthy babies and the second case left the hospital in good condition after three weeks in hospital.

Public health technicians of Bilbao council inspected the two shops indicated by the patients (shops A and B). On 30 August samples of the cheese were collected from shop B for laboratory investigations. After the first results which indicated the presence of Listeria, new triplicate samples were collected on 7 September according to the Spanish legislation.

Listeria was found in all the six samples collected of the cheese; five of those samples were fairly beyond the food safety criteria ( $(100 \mathrm{cfu} / \mathrm{g}$ ) [4,5] and one of them reached a value of $3.2 \times 10^{4} \mathrm{cfu} / \mathrm{g}$. All the strains isolated from the cheese in shop $B$ were characterised as $1 / 2$ a serotype. In shop $A$, where the first case used to buy this kind of cheese, no sample of the Latin-style fresh cheese was available during the inspection but it was proved that it had been commercialised there since April 2012.

The strains isolated both from the pregnant woman and the newborn and those isolated from cheese were sent to the National Reference Laboratory from the Institute of Health Carlos III, Madrid, for characterisation. These strains were analysed by PFGE, by digesting the DNA with Apal and following standardised protocols [6]. All the strains showed an indistinguishable pattern profile among them.

\section{Control measures}

After confirmation that the cheese was contaminated, an alert was issued via the Rapid Alert System for Food and Feed (RASFF) in Europe and the Public Health Department of the Basque Government ordered the withdrawal of the cheese from the market.

The great difference between the number of cheese packs distributed and those withdrawn from the market, and the long period until this cheese already sold reaches the expiry date (September or November), suggests that this product may still remain in several homes until November. Therefore, the Basque population was advised via mass media not to consume this type of cheese.
Health professionals in the Basque Country were informed about the situation and were recommended to strongly consider the diagnosis of listeriosis in highrisk individuals (pregnant or immunocompromised) with symptoms compatible with listeriosis and indicating having consumed Latin-style fresh cheese.

\section{Conclusion}

This report gives an indication that these two cases are not part of a longer ongoing outbreak and highlights that the investigation of every case of listeriosis is important even in a context of low incidence. Our report shows that a thorough epidemiological investigation allows a quick implementation of control measures that can prevent secondary cases.

Both cases described here were linked to the consumption of the same kind of cheese bought in stores selling Latin American specialities whose customers are mainly of Latin American origin. Outbreaks of listeriosis affecting pregnant Latin American women have been described before; in fact this group is described as a high-risk group because their consumption of fresh cheese is higher than that of people of other population groups $[7,8]$. The PFGE results and the results of the epidemiological investigation confirm the association between the listeriosis cases and the consumption of that particular type of cheese.

Taking into account the long incubation period of this illness, the widespread distribution of the cheese and its long period until reaching the expiry date, further cases may still occur although the cheese has been withdrawn from the market. So far, there have been no more cases in Bizkaia and we are not aware of any further cases having occurred in Spain or elsewhere.

Acknowledgments

Thanks to everyone who has taken active part in the present investigation: the staff of microbiology of the hospitals of Cruces and Basurto and the municipal laboratory of Bilbao, the epidemiologists of the Unit of Epidemiological Surveillance of Bizkaia, the technicians of Public Health in Food Safety Unit of Bizkaia and Uribe Kosta in the Basque Government, and to the subarea of Food Safety and Zoonosis of the Bilbao Council. 


\section{References}

1. Hof H. History and epidemiology of listeriosis. FEMS Immunol Med Microbiol. 2003; 35(3):199-202.

2. Mook P, Grant KA, Little CL, Kafatos G, Gillespie IA. Emergence of pregnancy-related listeriosis amongst ethnic minorities in England and Wales. Euro Surveill. 2010;15(27):pii=19610. Available from: http://www.eurosurveillance.org/ViewArticle. aspx?Articleld $=19610$

3. Dirección Territorial de Sanidad y Consumo de Bizkaia. Surveillance Report 2010. Unit of Epidemiological Surveillance. [Accessed: Sep 2012]. Available from: http://www.osakidetza. euskadi.net/r85pkvigio5/es/contenidos/informacion/ epidemiologica_publicaciones/es_epidemi/ro1hRedirectCont/ contenidos/informacion/epidemiologica_memorias/es epidemi/adjuntos/2010_bizkaia_completa.pdf

4. European Commission (EC). Commission Regulation (EC) No $2073 / 2005$ of 15 November 2005 on microbiological criteria for foodstuffs. EC; 2005. Official Journal of the European Union. Available from: http://eur-lex.europa.eu/LexUriServ/ LexUriServ.do?uri=0J:L:2005:338:0001:0026:EN:PDF

5. European Commission (EC). Commission Regulation (EC) No 1441/2007 of 5 December 2007 amending Regulation (EC) No 2073/2005 on microbiological criteria for foodstuffs. EC; 2007. Official Journal of the European Union. Available from: http:// eur-lex.europa.eu/LexUriServ/LexUriServ.do?uri=0J:L:2007:32 2:0012:0029:EN:PDF

6. Salcedo C, Arreaza L, Alcalá B, de la Fuente L, Vázquez JA. Development of a Multilocus Sequence Typing Method for Analysis of Listeria monocytogenes Clones. J Clin Microbiol. 2003;41(2):757-62.

7. Silk BJ, Date KA, Jackson KA, Pouillot R, Holt KG, Graves LM, et al. Invasive listeriosis in the Foodborne Diseases Active Surveillance Network (FoodNet), 2004-2009: further targeted prevention needed for higher-risk groups. Clin Infect Dis. 2012;54 Suppl 5:S396-404.

8. Pouillot R, Hoelzer K, Jackson KA, Henao OL, Silk BJ . Relative risk of listeriosis in Foodborne Diseases Active Surveillance Network (FoodNet) sites according to age, pregnancy, and ethnicity. Clin Infect Dis. 2012;54 Suppl 5:S405-10. 